\title{
Motor learning in children with developmental coordination disorder: The role of focus of attention and working memory
}

\author{
Sandra J.M. van Cappellen - van Maldegem ${ }^{\mathrm{a}, 1,2}$, Femke van Abswoude $^{\mathrm{a}, *, 1}$, \\ Hilde Krajenbrink ${ }^{\mathrm{a}}$, Bert Steenbergen ${ }^{\mathrm{a}, \mathrm{b}}$ \\ ${ }^{a}$ Behavioural Science Institute, Radboud University Nijmegen, Montessorilaan 3, PO Box 9104, 6500 HE Nijmegen, The Netherlands \\ ${ }^{\mathrm{b}}$ Centre for Disability and Development Research, Australian Catholic University, Melbourne, Australia
}

\section{A R T I C L E I N F O}

\section{Keywords:}

Motor learning

Developmental coordination disorder

Focus of attention

Working memory

\begin{abstract}
A B S T R A C T
Effective motor learning paradigms are essential for children with motor difficulties to enhance their motor skills and facilitate performance in physical activities and in daily life. This study aimed to examine the effect of feedback with an internal or external focus of attention on motor learning of children with probable Developmental Coordination Disorder (pDCD). In addition, the role of working memory capacity was examined. Children were recruited via physical therapists, who integrated the experimental procedures within therapy sessions. We analyzed data of 25 children between 5 and 11 years old. They practiced a novel motor task of throwing a 'slingerball' over three weeks, while receiving feedback with an internal or external focus of attention. Results showed that children improved throwing accuracy regardless of the type of feedback they received. Visuospatial working memory capacity enhanced learning, especially for children receiving feedback with an external focus of attention. These findings corroborate clinical recommendations stating that children with DCD benefit from task specific training and feedback, which is promoted with both foci of attention. However, the findings contrast the expected benefits of practice with an external focus of attention. It highlights that the exact mechanisms and task constraints that influence the learning processes with an internal and external focus among children are not yet understood and warrant further study.
\end{abstract}

\section{Introduction}

Children with Developmental Coordination Disorder (DCD) experience difficulties with learning and executing coordinated movements (Wilson et al., 2017). DCD is diagnosed in approximately 6\% of all children (DSM V, 2013. Compared with their typically developing peers, these children experience problems with activities of daily living such as using cutlery, tying shoes, catching a ball, riding a bike, and/or physical education (Zwicker, Harris, \& Klassen, 2013). Due to their motor limitations and resulting lower selfefficacy, children with DCD may avoid participation in free and organized play, and physical activities (Cairney et al., 2005; Fitzpatrick \& Watkinson, 2003). To facilitate participation in daily physical activities and society at large, it is warranted that effective and tailored training programs for motor learning are developed for these children (Farhat et al., 2016; Imms et al., 2017;

\footnotetext{
* Corresponding author.

E-mail address: f.vanabswoude@pwo.ru.nl (F. van Abswoude).

${ }^{1}$ These two authors contributed equally.

${ }^{2}$ Present address: Center of Research on Psychological and Somatic disorders, Tilburg University, PO Box 90153, 5000 LE Tilburg, The Netherlands.
} 
Smits-Engelsman et al., 2013).

Motor skills can be trained via more explicit or implicit motor learning paradigms. Explicit motor learning presumes that motor learning starts with an initial effortful, cognitive, and explicit stage and proceeds to a more effortless, autonomous, and implicit stage (Fitts \& Posner, 1967). Especially in the first stage of motor learning there is large cognitive involvement. In particular, the load on working memory is high as it is needed to hold and process incoming information, such as verbal instructions, visual feedback representing performance outcome, as well as proprioceptive and tactile sensory feedback resulting from the motor performance (Maxwell, Masters, \& Eves, 2003). Once the information is processed, this declarative knowledge can be used to create and test hypotheses on how movements can be performed more effectively (Masters, 1992). In contrast to explicit motor learning, implicit motor learning proposes that a learner can bypass the initial, effortful, cognitive stage, and directly start in to the effortless, autonomous stage of motor learning (Masters, 1992). As a result, participants who learned in an implicit manner do not accumulate declarative knowledge about movement execution and have, in fact, difficulties to articulate how they performed the movement (Masters 1992, also see Masters \& Poolton, 2012, for a review). Importantly, and in contrast to explicit motor learning, the involvement of working memory during the learning process is presumed to be reduced to a large extent (Masters, 1992; Steenbergen, van der Kamp, Verneau, Jongbloed-Pereboom, \& Masters, 2010).

A proportion of children with DCD are known to have co-morbid working memory problems (Alloway, 2007a, Piek, Dyck, Francis, \& Conwell, 2007, Wilson, Ruddock, Smits-Engelsman, Polatajko, \& Blank, 2013). These working memory problems are likely to play a role in the process of motor learning, in particular because working memory involvement is presumed to be a prerequisite for explicit motor learning (Maxwell et al., 2003). Working memory capacity is used to generate, store, and manipulate declarative knowledge and apply this to movement execution. As a result, it is expected that populations with reduced working memory capacity, like children with DCD, would benefit more from motor learning paradigms that promote implicit learning.

Several paradigms have been developed to promote implicit motor learning (Kleynen et al., 2015; Masters \& Poolton, 2012). In the present study we will use the paradigm of (re)directing the focus of attention of the learner to induce implicit or explicit motor learning. According to the constrained action hypothesis (Wulf, McNevin, \& Shea, 2001), an external focus of attention, viz. focusing on the movement outcome instead of the movement itself, will enhance automaticity of movement execution. This enhanced movement automaticity reduces working memory demands, since unconscious control processes predominantly regulate movement control. Thus, an external focus of attention is supposed to promote implicit motor learning (Kal, van der Kamp, \& Houdijk, 2013; Kleynen et al., 2015). In contrast, if the focus of attention is directed to body movements of the learner, viz. an internal focus of attention, explicit motor learning is supposed to be facilitated. An internal focus of attention proposes the involvement of working memory, because a conscious mode of control is triggered that interferes with the natural automatic control processes of movement execution (Wulf et al., 2001). Previous studies in adult populations have consistently shown that an external focus of attention leads to enhanced motor learning and performance over an internal focus of attention in a variety of motor tasks (see Wulf, 2013, for a review).

To validate the constrained action hypothesis the level of automaticity of a movement with an internal or external focus of attention is assessed via the dual task paradigm (Kal et al., 2013; Poolton, Maxwell, Masters, \& Raab, 2006; Wulf et al., 2001). If the movement is executed automatically, less cognitive resources are needed to control the movement, and thus a secondary task can be performed without interference with the primary motor task (see Abernethy, 1988, and Houwink, Steenbergen, Buurke, Pranke, \& Geurts, 2013). In line with the constrained action hypothesis, several studies showed that movements with an internal focus of attention were indeed hindered by the simultaneous execution of a secondary task parallel to the motor task. It was also shown that movements performed with an external focus of attention were not hindered by this secondary task loading (Kal et al., 2013; Poolton et al., 2006; Wulf et al., 2001). These collective findings exemplify that an external focus of attention leads to more automatization of the movements, thereby decreasing working memory demands.

The lower working memory demands that are associated with motor learning using an external focus of attention makes it potentially suitable for individuals with a reduced working memory capacity, such as children with DCD. Nonetheless, up to date, this was only tested in three studies, with varying results. Chiviacowsky, Wulf, and Ávila (2013) studied children with mild intellectual disabilities, Saemi, Porter, Wulf, Ghotbi-Varzaneh and Bakhtiari (2013) studied children with ADHD, and Jarus et al. (2015) studied motor learning in children with DCD. While Chiviacowsky et al. (2013) and Saemi et al. (2013) showed a beneficial effect of an external focus of attention over an internal focus of attention, Jarus et al. (2015) did not find a significant difference between an internal and external focus of attention. Chiviacowsky et al. (2013) argued that the findings in favour of an external focus of attention were possibly caused by a potential freeing up of attentional capacity that may have resulted in the learning advantages. However, they did not directly test working memory involvement in the participating children. In sum, the results of existing studies concerning foci of attention in atypically developing children are equivocal, but it was suggested in these studies that the findings may be related to the attentional capacity of the participant (Chiviacowsky et al., 2013; Wulf \& Prinz, 2001). Therefore, in the present study the mediating role of working memory capacity on motor learning will be directly tested.

The main aim of the present study was to examine the effects of feedback promoting an internal or external focus of attention on motor learning in children with probable DCD (pDCD). To this end, we utilized a motor task that was new for the children (i.e., throwing a 'Slingerball') to ensure that they started in the first, cognitive stage of motor learning. We hypothesized that children in the external focus of attention group would show superior learning on the Slingerball throwing task (i.e., increased throwing accuracy) as compared to children in the internal focus of attention group. As a secondary aim we examined the mediating role of working memory capacity on learning with an internal (i.e. explicit learning) or external (i.e. implicit learning) focus of attention. 


\section{Methods}

\subsection{Participants}

Children (4-12 years) were recruited at private paediatric physiotherapy practices by 12 participating physical therapists. Together they recruited 35 children that were eligible to participate in this study based on a suspicion of DCD. Children were assessed on the Movement Assessment Battery for Children-2 (MABC-2) and if they scored lower or equal to the 16th percentile they were included in the study (DSM-V, criterion A). Seven children were excluded based on a score on the MABC-2 higher than the 16th percentile. Following the assessments, two children were excluded because they had too much missing data on either the pretest or posttest. This yielded an experimental group $(N=26)$ consisting of 23 boys and 3 girls with a mean age of 6.92 years old $(S D=1.70)$ at the onset of the study. Criterion B of the DSM-V (motor problems interfere with daily life activities) was assumed to be met as children were treated by a physical therapist. In addition, Criterion $\mathrm{C}$ of the DSM-V about early onset of symptoms is present since the children in the study are between 4 and 12 years of age. Finally, all children attended mainstream primary education, inferring an IQ > 70 (criterion D). Other neurological conditions or comorbidities were not registered. Parents of the children provided written informed consent for participation of their children in the study. Additionally, the study was approved by the local Ethics Review Committee (EC2013-1811-147a1).

\subsection{Background variables}

\subsubsection{Working memory capacity}

Working memory capacity was assessed by use of the Automated Working Memory Assessment (AWMA), which is a computerized tool for individuals aged 4-22 years (Alloway, 2007b). Two subtests assessing verbal working memory and visuospatial working memory (i.e., listening recall and spatial recall, respectively) were used. In the listening recall, children listened to a series of short sentences. After each sentence they needed to indicate if the sentence was true or false. At the end of the series, children had to recall the first word of each sentence in the correct order. The test started with one sentence and word to recall. After four correct trials, one sentence was added and this continued until children were unable to correctly recall the words on four out of six trials. The procedure was similar in the spatial recall, but instead of hearing sentences, children now had to look at shapes. In each trial children were shown a series of paired shapes, one of these shapes featured a dot and could be rotated and/or be the mirror image. After each pair, children had to indicate whether the shape with the dot was the same or the mirror image of the shape next to it. At the end of the series, they had to recall the position of the dots in the correct order. Only the memory scores on the working memory tests were used in further analyses. Test-retest reliability of these subtest was good with reliability coefficients of 0.88 and 0.79 (Alloway, $2007 \mathrm{~b}$ ).

\subsubsection{Motor skills}

Motor skills were determined based on the outcomes on the Movement Assessment Battery for Children - second edition (MABC2; Henderson, Sugden, Barnett, \& Smits-Engelsman, 2010). The MABC-2 consists of three main components assessing manual dexterity, catching and throwing, and balance. At the end of the test standard scores for the three domains and the total test were converted to percentiles.

\section{3. 'Slingerball' throwing task}

The experimental task was similar to the one used by Krajenbrink, van Abswoude, Vermeulen, van Cappellen, \& Steenbergen (2018). Children were instructed to hold the ribbon and to swing the 'slingerball' (see Fig. 1). The child had to release the 'slingerball' at the right moment, in order for it to land on the target area. The horizontal target area was a circle with a diameter of $2 \mathrm{~m}$, that consisted of eight concentric circles of a width of $12.5 \mathrm{~cm}$ each. This allowed for consistent scoring of accuracy of the throw. The bull's eye had a radius of $12.5 \mathrm{~cm}$ and the concentric circles had radii of $25,37.5,50,62.5,75,87.5,100 \mathrm{~cm}$. Points were assigned to

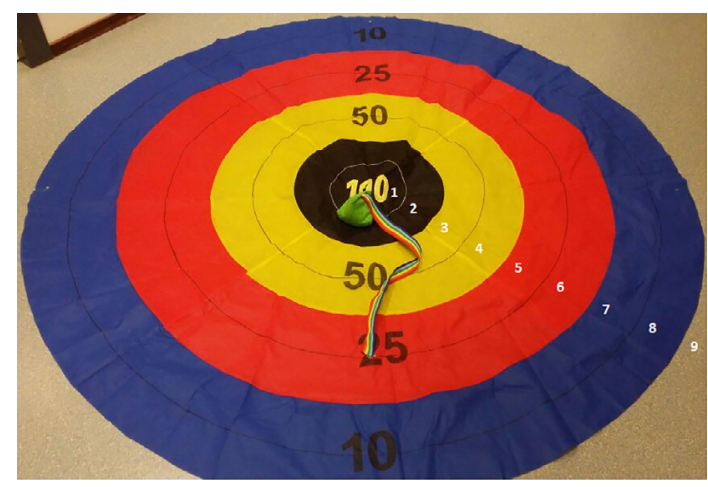

Fig. 1. Scoring of accuracy on the target area. 
Day 1

Pretest:
20 throws
Feedback
Followed by
practice (total
70 throws):
10 throws
Break
20 throws
Feedback
10 throws
Break
20 throws
Feedback
10 throws

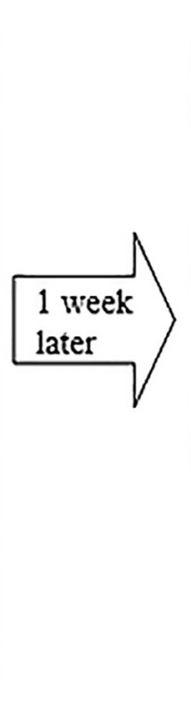

Day 2

Day 3

\section{Posttest:} 20 throws

\section{Feedback}

Feedback

10 throws

Break

20 throws

Feedback

10 throws
AWMA

MABC-2 (for children with no recent scores)

Fig. 2. Overview of the experimental procedures.

these zones: 1 (=)bull's eye), 2, 3, 4, 5, 6, 7, 8, or 9 (=outside the circles) points (see Fig. 1). Distance of the child to the target area was set at $5 \mathrm{~m}$.

\subsection{Procedure}

The study was a quasi-experimental field-based study using a pre-posttest design. We formed a partnership with local physiotherapists that allowed us to integrate testing and training of the 'slingerball' throwing task into physiotherapy sessions, thereby ensuring a familiar environment for the children. The physiotherapists were randomly assigned to a group providing either feedback with an internal focus of attention or an external focus of attention. This procedure prevented that therapists had to provide both types of feedback to different children, which would increase the risk of confusion or preferences of the therapists. In a meeting prior to the start of the training sessions physiotherapists were instructed on how to provide feedback according their designated focus of attention.

In three sessions, across a three-week period, each child completed the pretest and the first practice session in the first week, the second practice session in the second week, and the posttest in the third week (see Fig. 2). Before the pretest, all children received the same standardized instructions from their therapist on how to perform the 'slingerball' throwing task (see Appendix A). During practice, children received feedback with either an internal focus of attention (e.g., 'ensure your arm is higher when you let loose') or an external focus of attention (e.g., 'ensure the ball is higher when you let it go') at predetermined moments. A standardized protocol for the physical therapists, specifically describing the feedback to be provided to the children for the corresponding focus of attention, was used (see Appendix A). Specifically, during each feedback moment there was only one predetermined performance aspect on which children received feedback.

The posttest, during which no feedback or instructions were given, was directly followed by the assessment of working memory (AWMA). All children were individually tested in a quiet room at the physiotherapy practice. In case no recent MABC-2 scores of the child were available (i.e., assessed less than three months ago), the MABC-2 was repeated to assess current motor skills of the child.

\subsection{Statistical analysis}

Throwing accuracy on the pretest and posttest was determined by averaging the scores across the 20 trials for both phases. A 2 (focus: internal vs. external) x 2 (test: pretest vs. posttest) analysis of variance (ANOVA) with repeated measures on the last factor was preformed to analyse the difference in learning between groups. In addition, the role of working memory capacity on learning was determined with two separate linear regression models. To this end, learning was defined as the difference in throwing accuracy between the pretest and the posttest. Also, the scores on both working memory tests were standardized by subtracting the mean of the variable and dividing this by the standard deviation, creating the z-score. The first model focussed on visuospatial working memory and included pretest accuracy, focus of attention, visuospatial working memory, and the interaction between visuospatial working memory and focus of attention as independent variables, with learning as the dependent variable. The second regression model focussed on verbal working memory and included the predictors pretest accuracy, focus of attention, verbal working memory, and the interaction between verbal working memory and focus of attention, again with learning as the dependent variable. 
Table 1

Description of the participants.

\begin{tabular}{|c|c|c|c|c|c|}
\hline & & External focus & Internal focus & $t$ & Cohen's $d$ \\
\hline \multicolumn{6}{|l|}{ Participants (n) } \\
\hline & Boys & 10 & 12 & & \\
\hline & Girls & 3 & 0 & & \\
\hline Age in years & & $7.31(1.90)$ & 6.75 (1.09) & 0.86 & 0.35 \\
\hline MABC-2 percentile & & $2.78(2.93)$ & $4.63(3.51)$ & -1.38 & 0.55 \\
\hline \multirow[t]{2}{*}{ Working memory capacity } & Verbal & $10.00(4.08)$ & $8.92(4.05)$ & -0.43 & 0.17 \\
\hline & Visuospatial & $11.08(4.98)$ & $11.92(4.31)$ & 0.64 & 0.26 \\
\hline Pretest throwing accuracy & & $8.30(0.62)$ & $7.53(0.83)$ & $2.16^{*}$ & 1.05 \\
\hline Posttest throwing accuracy & & $7.86(1.12)$ & $7.22(0.87)$ & 1.58 & 0.64 \\
\hline
\end{tabular}

Note: values represent means and standard deviations unless otherwise defined.

* Indicates a significant difference, $p<.05$.

Statistical significance was set at $\mathrm{p}<.05$. For the partial eta squared effect sizes, 0.01 was considered a small effect, 0.06 was considered a medium effect, and 0.14 was considered a large effect (Cohen, 1988). Data analysis was performed in SPSS version 25.

\section{Results}

\subsection{Description of groups}

Exploration of the data showed that one participant was an univariate outlier ( $\pm 3 *$ SD on the change in throwing accuracy) and was therefore removed from the analysis. The remaining group thus consisted of 25 children, 22 boys and 3 girls, with a mean age of 7.08 years old $(S D=1.64)$ at the onset of the study. The internal and external focus of attention groups did not significantly differ on MABC-2 percentile scores, AWMA scores, or mean age (see Table 1). Independent sample $t$-test showed that there was a significant difference between both groups on pretest throwing accuracy $(t(23)=-2.62, p=.015)$. Specifically, children in the internal focus of attention group threw closer to the target compared to the children in the external focus of attention group (see Fig. 3 ).

\subsection{Learning effect}

The results of the repeated measures ANOVA showed a main effect of test, $F(1,23)=5.88, p=.024, \eta_{p}{ }^{2}=0.20$, indicating that children improved their throwing accuracy from pretest $(M=7.93, S D=0.80)$ to posttest $(M=7.55, S D=1.02)$. There was also a significant main effect for focus, $F(1,23)=4.94, p=.036, \eta_{p}{ }^{2}=0.18$, showing that children receiving an internal focus of attention $(M=7.38, S D=0.83)$ threw more accurate compared to children receiving an external focus of attention $(M=8.08, S D=0.90)$. Finally, the interaction between test and focus was not significant indicating that the improvement from pretest to posttest was similar for both groups (see Fig. 3).

Focusing in more detail on the individual change in throwing accuracy from pretest to posttest, we found that 17 children (68\%) improved their accuracy with an average of 0.7 (range: $0.05-1.75$ ). The accuracy of 7 children (32\%) declined with an average of 0.4 (range: 0.03-1.00), and the accuracy of one child remained exactly the same. Out of the 17 children that improved their accuracy, 9 children received an internal focus of attention and 8 children received an external focus of attention. The number of children that reduced their accuracy was also equally distributed over both groups; 3 children with an internal focus and 4 children with an external focus showed a decline.

\subsection{Role of working memory on learning}

The regression model including the variables pretest accuracy, visuospatial working memory, focus of attention, and the interaction between visuospatial working memory and focus of attention led to a significant model predicting learning $(F(4,20)=3.609$, $p=0.023$, adjusted $\left.R^{2}=0.303\right)$. It further showed that the accuracy in the pretest had no significant effect on learning $(\beta=-0.10$, $p=.627)$. Also, there was no significant effect of focus of attention $(\beta=0.08, p=.785)$. The results further showed a significant effect of visuospatial working memory on learning $(\beta=-1.45, p<.01)$. Children with better visuospatial working memory capacity improved their accuracy more than children with lower visuospatial working memory capacity. Finally, a significant interaction was found between visuospatial working memory capacity and focus of attention $(\beta=0.85, p<.01)$. This interaction was followed-up by calculating simple slopes separately for the internal and external focus of attention groups. This analysis indicated that children receiving feedback with an external focus of attention improved more if they had a better visuospatial working memory capacity $(b=-1.45, s e=0.40, p<.01)$. For children receiving feedback with an internal focus of attention the relation between visuospatial working memory capacity was not significant $(b=-0.60$, se $=0.17, p>.05$, see Fig. 4 for the relation between visuospatial working memory and learning in both groups). The regression model including verbal working memory revealed no significant effects $\left(F(4,20)=0.438, p=.78\right.$, adjusted $\left.R^{2}=-0.103\right)$. 


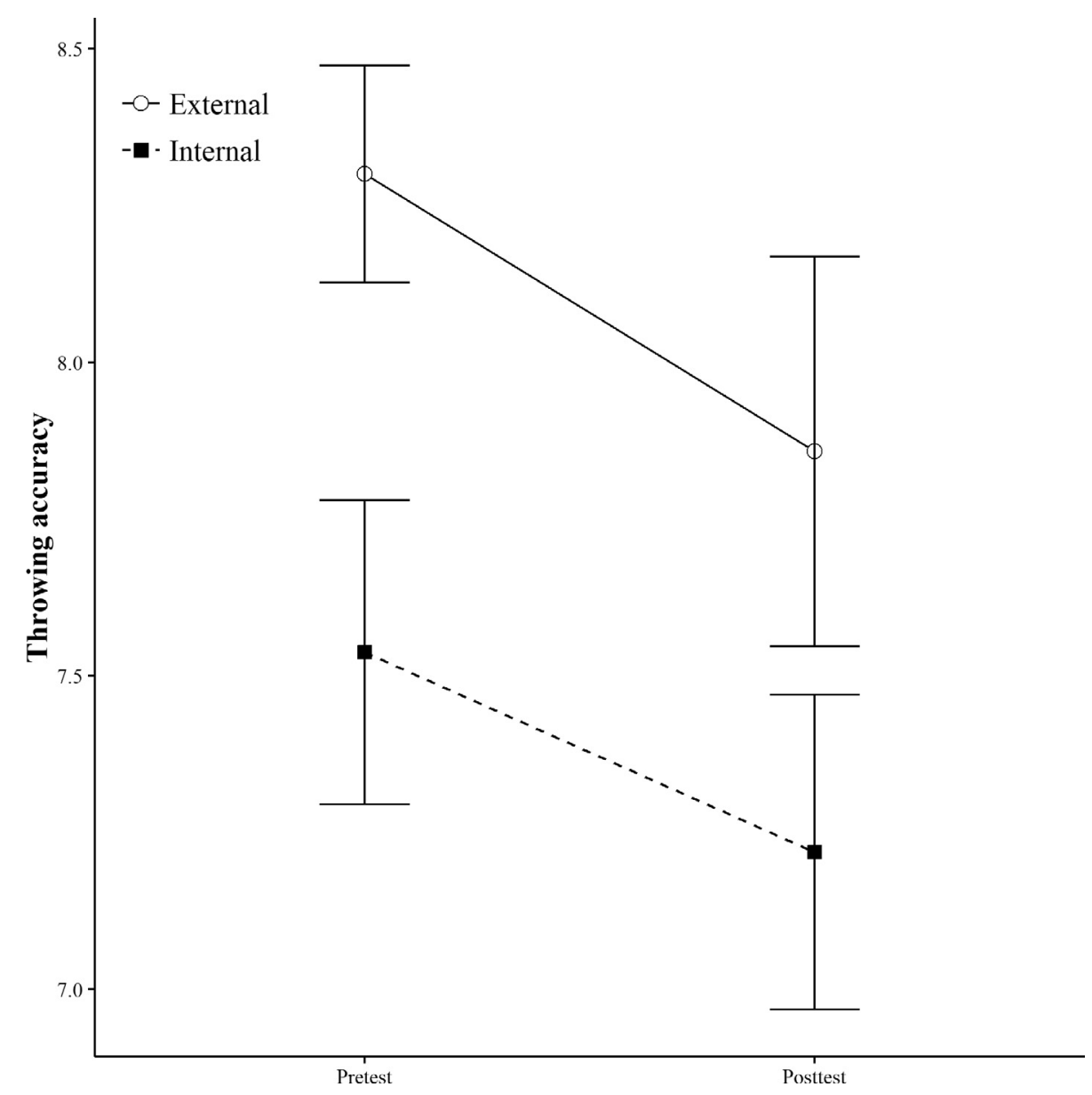

Fig. 3. Throwing accuracy on the pretest and posttest in both groups (error bars represent standard error).

\section{Discussion}

In the current study we examined the effect of feedback with either an internal or external focus of attention on motor learning of children with pDCD. The results show that children with pDCD improved their performance with respect to throwing accuracy on the 'slingerball' task with both types of feedback. However, contrary to our expectation we did not find differential effects of focus of attention on learning. In addition, our results showed that visuospatial working memory capacity had an effect on motor learning with an external but not an internal focus of attention. More specifically, children receiving an external focus of attention improved more when they also had better visuospatial working memory capacity. For children receiving an internal focus of attention there was no effect of working memory capacity on motor learning.

This is the first study that examines the effect of feedback with either an internal or external focus of attention on a novel, complex motor task in a group of children with pDCD. A priori, we hypothesized a beneficial effect of an external focus, but our results are in line with the study of Jarus et al. (2015) who also failed to show any differences in motor learning with an internal or external focus of attention in children with DCD using a computerized tracking task. These findings suggest that practice itself, regardless of the attentional focus, contributes to improvements in performance in children with pDCD. This is in line with the recommendations regarding motor learning in children with DCD (Schoemaker \& Smits-Engelsman, 2015; Smits-Engelsman et al., 2013) which state that task specific training is the most important factor leading to improvements in performance. Even though not all children in the current study had the formal clinical diagnosis of DCD, the motor problems of the children were profound enough to be treated by a physical therapist. Thus, although our sample can be characterized as children with pDCD, it also carries close resemblance to the clinically diagnosed children with DCD (Geuze, Schoemaker, \& Smits-Engelsman, 2015). Our finding that these children can actually benefit from both types of feedback for motor learning suggests that these types of feedback are suitable to be used in clinical practice to train motor skills in children with DCD.

In the current study the feedback was provided by the children's physical therapists which highlights the potential applicability of our findings. For reasons of reproducibility we used a standardised protocol that did not allow for individualized feedback. Irrespective thereof, the children did learn following the practice sessions. We anticipate that a further adaptation of the content and frequency of feedback to the individuals needs and preferences may elicit even larger improvements and could lead to more 


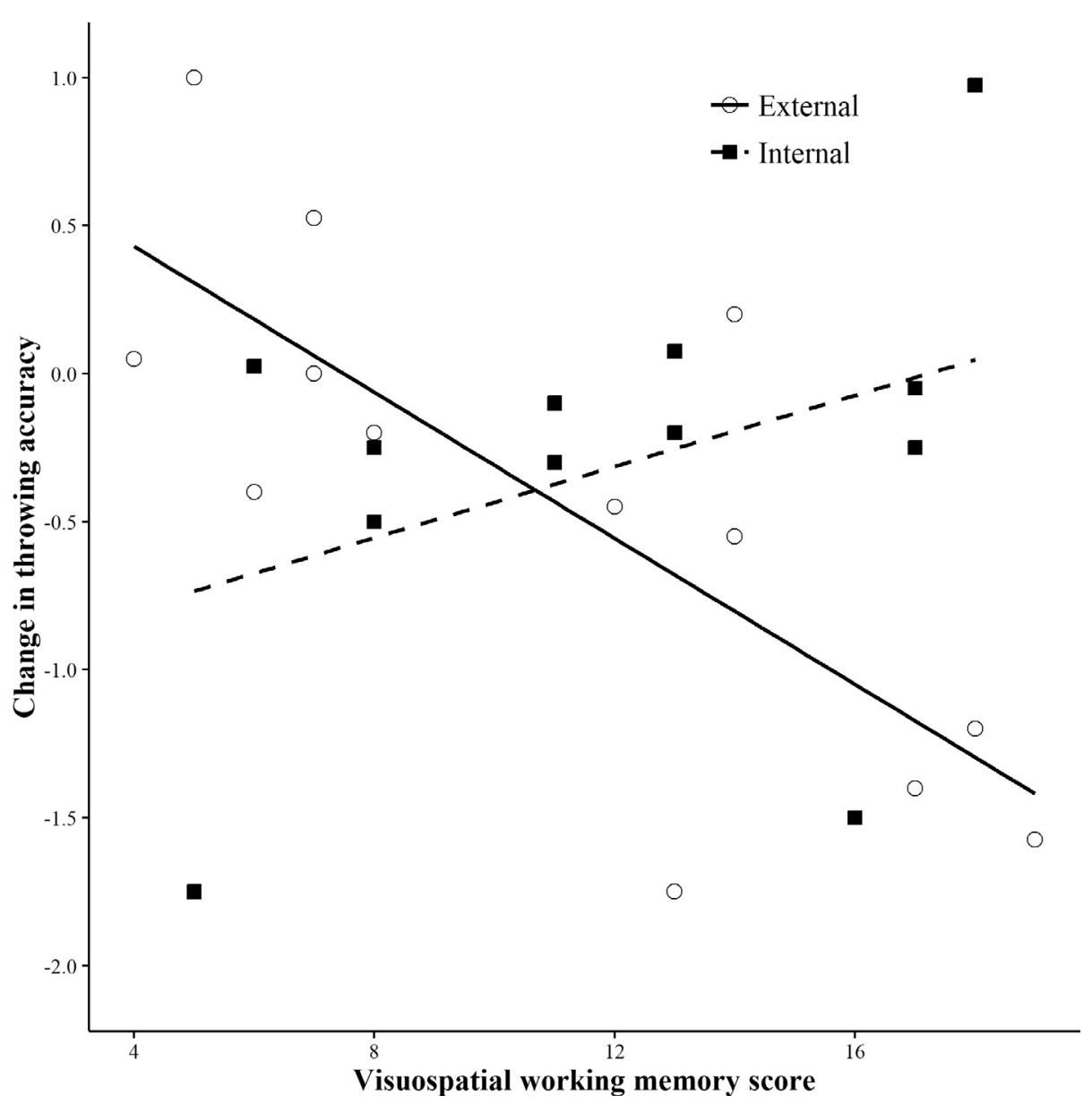

Fig. 4. Graph visualizing the correlation between visuospatial working memory scores and received focus of attention in relation to learning (internal focus: $r=0.38$; external focus: $r=-0.76$ ). A negative value indicates an improvement in throwing accuracy.

pronounced differences between both types of attentional foci. As an illustration, in the study of Wulf, Chiviacowsky, Schiller and Ávila (2010), feedback with an external focus was most beneficial when it was provided after every trial. More frequent feedback and feedback directed at specific elements of the individual's performance that needed improvement might lead to differences between the groups.

The high ecological validity of the current study was inevitably somewhat at the cost of experimental control. During the experiment, therapists provided the feedback to create the most natural environment for the children. In addition, to prevent additional pressure for the children, experimenters were not present during the practice phase. Unfortunately, we were not able to check whether all therapists applied the protocol as discussed in the meeting prior to the start of the study. In addition, we did not record the other activities that were undertaken in the therapy sessions during the study period. It is possible that therapists adjusted their activities and practiced aiming and catching skills which could lead to the 'training to the test' phenomenon. However, by using the 'slingerball', which requires a different coordination pattern compared to 'regular' aiming activities, we are confident to have minimized this effect. Finally, instead of randomising participants, therapists were randomly assigned to the groups. That way, therapists only had to provide one type of feedback to all children. It should be mentioned that this is a limitation as therapists might differ with regard to their characteristics (e.g., experience, quality), their practices (e.g., treating children with more profound or milder motor problems) and their possible feedback preferences. However, by comparing the participating children on motor skills and working memory capacity, we did control for possible differences in population between the groups. We did see differences in performance on the pretest between the groups. Nevertheless, improvements were similar in both groups and the general level of motor skills was not significantly different, suggesting that a slight difference in motor skills might have influenced the initial performance of children, but not their motor learning ability.

Our results regarding working memory are not in line with the theory of explicit motor learning and the constrained action hypothesis (Maxwell et al., 2003; Wulf et al., 2001). Both theories predict that practice with an internal focus of attention would lead to a higher degree of cognitive control and we expected that this would lead to a larger involvement of cognitive resources compared to practice with an external focus of attention. While these theories do not specify which cognitive resources are employed, literature expects a role of verbal working memory capacity to cope with these verbal instructions and apply them to movement execution 
(Buszard, Masters, \& Farrow, 2017; Buszard \& Masters, 2017; Maxwell et al., 2003). Our results showed that visuospatial working memory capacity, and not verbal working memory, had an effect on motor learning. This effect was only present for the group that practiced with an external focus of attention.

This unexpected role for working memory in motor learning may be related to the nature of the new motor task as it requires a complex coordination of the arm in combination with the movement of the 'slingerball' itself. This requires a translation of the spatial coordinates and kinematic information of the arm, ribbon, ball, and target to actual motor performance, which may be more depended on visuospatial working memory capacity (Quinn 2008). We can only speculate why the relation between learning and visuospatial working memory capacity was only present in the group receiving feedback with an external focus. It may be that children with (p)DCD are more familiar with an internal focus of attention. Research already showed that therapists working with adults predominantly use instruction with an internal focus of attention (Kal et al., 2017). If this is also the case for therapists working with children, this may cause the children to prefer a habitual internal focus of attention that focuses on their movement coordination. Receiving feedback with an unfamiliar or non-preferred external focus may be less optimal (Maurer \& Munzert, 2013; van Abswoude, Nuijen, van der Kamp, \& Steenbergen, 2018), thereby placing a higher demand on working memory capacity. Matching instructions or feedback to the preference or familiarity with an attentional focus may also enhance learning in general. Finally, we expected children with pDCD to show lower working memory capacity compared to their typically developing peers (Alloway, 2007a, Piek et al., 2007, Wilson et al., 2013). However, the range in working memory capacity of our participants as a group is comparable to that of typically developing children in the same age range (Alloway, Gathercole, \& Pickering, 2006). This could partially explain why we did not show the expected benefit of an external focus of attention for children with pDCD.

While we found a role for visuospatial working memory capacity in the external focus group, it would be premature to conclude that the external focus led to an explicit learning process or consciously controlled movement execution. More measures that could elucidate if movement automatization differs between groups are needed to draw firm conclusions. For example, by including a dual task to 'stress' working memory capacity (e.g., Krajenbrink et al., 2018) or by measuring brain activity to study which regions are activated during movement production (e.g., Buszard, Farrow, Zhu, \& Masters, 2016).

The current findings add important evidence to the increasing body of literature showing a lack, or an unexpected role of working memory for motor learning of children (Brocken, Kal, \& van der Kamp, 2016; Jongbloed-Pereboom, Peeters, Overvelde, Nijihuis-van der Sanden, and Steenbergen (2015); Krajenbrink et al., 2018; van Abswoude, Nuijen et al., 2018; van Abswoude, van der Kamp, \& Steenbergen, 2018). For example, in a recent study, van van Abswoude, van der Kamp et al., 2018 found only a small effect of visuospatial working memory capacity on performance during practice of an aiming task in an explicit context. However, this effect was not present for retention one day later. Brocken et al. (2016) also failed to show a role for working memory capacity on learning with an internal or external focus of attention, although they only investigated verbal working memory. Finally, in the studies of Krajenbrink et al. (2018) and van van Abswoude, Nuijen et al. (2018) there was also no role of working memory capacity for respectively learning over a one week period, and performance during practice, with internal or external focus instructions.

In sum, our study did not find the expected differences in learning after receiving feedback with an internal or external focus of attention, although, as a general rule, the children with pDCD did improve their aiming accuracy. To our knowledge only two studies did show a difference between learning with an internal or external focus of attention in children with a-typical development (Chiviacowsky et al., 2013; Saemi et al., 2013). However, retention periods for these studies were shorter, $24 \mathrm{~h}$ and $48 \mathrm{~h}$ respectively. Also in typically developing children, benefits of an external focus of attention were only found during or immediately after practice (Abdollahipour, Wulf, Psotta, \& Nieto, 2015, Krajenbrink et al., 2018) or after a 24 h to 48 h delay (Brocken et al., 2016; Flores, Schild, \& Chiviacowsky, 2015; Hadler, Chiviacowsky, Wulf, \& Schild, 2014; Thorn, 2006). Moreover, some studies did not find any differences between both attentional foci either during practice or after a short retention (Chow, Koh, Davids, Button, \& Rein, 2014; Emanuel, Jarus, \& Bart, 2008; Perreault \& French, 2016; van Abswoude, Nuijen et al., 2018) or after a longer retention period (Krajenbrink et al., 2018). Thus, the evidence with respect to the proposed beneficial effects of an external focus for motor learning in children is presently inconclusive at best and seems to be short lived. The diversity in methodology (i.e., task complexity, instructional content, etc.) and participants in the studies (e.g., age, typical or a-typical development, experience) makes it difficult to compare these outcomes. Also, the role of individual differences (e.g., cognitive capacities, motor skills, preferences) and the actual learning process is not yet understood. In the present study we did not register the performance during practice. We suggest that future studies do take this into account to create individual learning curves and to determine if an external focus is perhaps more beneficial at an earlier stage. Taken together, more systematic research into the constraints that influence the effect of attentional focus on motor learning in children is warranted in order to make well informed recommendation for interventions.

\section{Conclusion}

This study showed that children with pDCD learned a novel complex motor task while receiving feedback with both an internal and an external focus of attention. These findings correspond with the clinical recommendations for children with DCD that highlight task specific instructions and practice to improve motor performance. We also showed that children with better visuospatial working memory capacity improved to a larger extent, especially when practising with an external focus of attention. The findings contrast both the beneficial role of an external focus of attention and the independence of cognitive capacities. It adds to the body of literature indicating that the effect of attentional focus on motor learning in children is different compared to the expected effect based on motor learning in adults. Nevertheless, our results suggest that task-specific feedback during practice can improve task performance in children with profound motor difficulties, which is promising for clinical practice. Clearly, the research into the effects of an internal and external focus of attention and the task constraints that affect its impact is an important area that warrants further study. 


\section{Declarations of interest}

None.

\section{Funding}

This work was supported by the Nederlandse Organisatie voor Wetenschappelijk Onderzoek (NWO; project 328-98-004).

\section{Acknowledgement}

The authors wish to thank all the physical therapists, children and their parents for participating in this study. This work was supported by the Nederlandse Organisatie voor Wetenschappelijk Onderzoek (NWO; project 328-98-004).

\section{Appendix A:. Instructions and feedback used in the experiment}

\section{General instructions of the 'slingerball' task}

The physical therapists gives the following instruction to all children:

"In this exercise you are going to aim this 'slingerball' at the target. You can swing the ball around a few times before you let it go".

This instruction is followed by one demonstration.

\section{Feedback}

In total there are 5 moments that the child received feedback with a specific focus of attention. The order of this feedback was standardized.

\section{Internal focus}

1. Make sure your arm is stretched less/more when you swing the ball.

2. Make sure your arm is lower/higher when you release it.

3. Make sure your arm turns slower/faster before you let go.

4. Make sure your arm is lower/higher when you release it.

5. Make sure you let go sooner/later.

\section{External focus}

1. Make sure the ribbon is slacker/tighter when you swing it.

2. Make sure the ball is lower/higher when you let it go.

3. Make sure the ball turns slower/faster before you let it go.

4. Make sure the ball is lower/higher when you let it go.

5. Make sure you let it go sooner/later.

\section{References}

Abdollahipour, R., Wulf, G., Psotta, R., \& Nieto, M. P. (2015). Performance of gymnastics skill benefits from an external focus of attention. Journal of Sports Sciences, 33(17), 1807-1813. https://doi.org/10.1080/02640414.2015.1012102.

Abernethy, B. (1988). Dual-task methodology and motor-skills research - Some applications and methodological constraints. Journal of Human Movement Studies, 14(3), 101-132.

Alloway, T. P. (2007a). Working memory, reading, and mathematical skills in children with developmental coordination disorder. Journal of Experimental Child Psychology, 96, 20-36. https://doi.org/10.1016/j.jecp.2006.07.002.

Alloway, T. P. (2007b). Manual automatized working memory assessment. Oxford: Pearson.

Alloway, T. P., Gathercole, S. E., \& Pickering, S. J. (2006). Verbal and visuospatial short-term and working memory in childre: Are they seperable? Child Development, 77(6), 1698-1716. https://doi.org/10.1111/j.1467-8624.2006.00968.x.

American Psychiatric Association (2013). Diagnostic and statistical manual of mental disorders (DSM-5®). American Psychiatric Pub.

Brocken, J. E. A., Kal, E. C., \& van der Kamp, J. (2016). Focus of attention in children's motor learning: Examining the role of age and working memory. Journal of Motor Behavior, 48, 527-534. https://doi.org/10.1080/00222895.2016.1152224.

Buszard, T., Farrow, D., Zhu, F., \& Masters, R. S. W. (2016). The relationship between working memory capacity and cortical activity during performance of a novel motor task. Psychology of Sport and Exercise, 22, 247-254. https://doi.org/10.1016/j.psychsport.2015.07.005.

Buszard, T., \& Masters, R. S. (2017). Adapting, correcting and sequencing movements: Does working-memory capacity play a role? International Review of Sport and Exercise Psychology, 11(1), 1-21. https://doi.org/10.1080/1750984X.2017.1323940.

Buszard, T., Masters, R. S. W., \& Farrow, D. (2017). The generalizability of working-memory capacity in the sport domain. Current Opinion in Psychology, 16, 54-57. https://doi.org/10.1016/j.copsyc.2017.04.018.

Cairney, J., Hay, J. A., Faught, B. E., Wade, T. J., Corna, L., \& Flouris, A. (2005). Developmental coordination disorder, generalized self-efficacy toward physical activity, and participation in organized and free play activities. The Journal of pediatrics, 147(4), 515-520. https://doi.org/10.1016/j.jpeds.2005.05.013.

Chiviacowsky, S., Wulf, G., \& Ávila, L. T. G. (2013). An external focus of attention enhances motor learning in children with intellectual disabilities. Journal of Intellectual Disability Research, 57(7), 627-634. https://doi.org/10.1111/j.1365-2788.2012.01569.x.

Chow, J. Y., Koh, M., Davids, K., Button, C., \& Rein, R. (2014). Effects of different instructional constraints on task performance and emergence of coordination in 
children. European Journal of Sport Science, 14(3), 224-232. https://doi.org/10.1080/17461391.2013.780097.

Cohen, J. (1988). Statistical power analysis for the behavioral sciences (2nd ed.). Hillsdale, N.J: Lauwrence Erlbaum Associates.

Emanuel, M., Jarus, T., \& Bart, O. (2008). Effect of focus of attention and age on motor acquisition, retention, and transfer: A randomized trial. Physical Therapy, 88(2), 251-260. https://doi.org/10.2522/ptj.20060174.

Farhat, F., Hsairi, I., Baati, H., Smits-Engelsman, B. C. M., Masmoudi, K., Mchirgui, R., ... Moalla, W. (2016). The effect of a motor skill training program on the improvement of practiced and non-practiced task performance in children with developmental coordination disorder (DCD). Human Movement Science, 46, 10-22. https://doi.org/10.1016/j.humov.2015.12.001.

Fitts, P. M., \& Posner, M. I. (1967). Human performance. Belmont, CA: Brooks/Cole.

Fitzpatrick, D. A., \& Watkinson, E. J. (2003). The lived experience of physical awkwardness: Adults' retrospective views. Adapted Physical Activity Quarterly, 20(3), 279-297. https://doi.org/10.1123/apaq.20.3.279.

Flores, F. S., Schild, J. F. G., \& Chiviacowsky, S. (2015). Benefits of external focus instructions on the learning of a balance task in children of different ages. International Journal of Sport Psychology, 46(4), 311-320. https://doi.org/10.7352/Ijsp2015.46.311.

Geuze, R. H., Schoemaker, M. M., \& Smits-Engelsman, B. C. M. (2015). Clinical and research criteria for developmental coordinatio disorder - should they be one and the same? Current Developmental Disorders Reports, 2(2), 127-130. https://doi.org/10.1007/s40474-015-0043-9.

Hadler, R., Chiviacowsky, S., Wulf, G., \& Schild, J. F. G. (2014). Children's learning of tennis skills is facilitated by external focus instructions. Motriz: Revista de Educação Física, 20, 418-422.

Henderson, S. E., Sugden, D. A., Barnett, A. L., \& Smits-Engelsman, B. C. M. (2010). Movement assessment battery for children - second edition, Dutch translation. Amsterdam: Pearson Assessment and Information B.V.

Houwink, A., Steenbergen, B., Buurke, J., Pranke, G., \& Geurts, A. C. H. (2013). Upper-limb motor control in patients after stroke: Attentional demands and the potential beneficial effects of gravity support. Human Movement Science, 32(2), 377-387. https://doi.org/10.1016/j.humov.2012.01.007.

Imms, C., Granlund, M., Wilson, P. H., Steenbergen, B., Rosenbaum, P. L., \& Gordon, A. M. (2017). Participation, both a means and an end: A conceptual analysis of processes and outcomes in childhood disability. Developmental Medicine and Child Neurology, 59(1), 16-25. https://doi.org/10.1111/dmcn.13237.

Jarus, T., Ghanouni, P., Abel, R. L., Fomenoff, S. L., Lundberg, J., Davidson, S., ... Zwicker, J. G. (2015). Effect of internal versus external focus of attention on implicit motor learning in children with developmental coordination disorder. Research in Developmental Disabilities, 37, 119-126. https://doi.org/10.1016/j.ridd.2014.11. 009.

Jongbloed-Pereboom, M., Peeters, A., Overvelde, A., Nijihuis-van der Sanden, M. W. G., \& Steenbergen, B. (2015). Learning of writing letter-like sequences in children with physical and multiple disabilities. Research in Developmental Disabilities, 36, 150-161. https://doi.org/10.1016/j.ridd.2014.10.005.

Kal, E., van den Brink, H., van der Kamp, J., Goossens, P., van Bennekom, C., \& Scherder, E. (2017). How physical therapists instruct patients with stroke: An observational study on attentional focus during gait rehabilitation after stroke. Disability and Rehabilitation. https://doi.org/10.1080/09638288.2017.1290697.

Kal, E. C., Van der Kamp, J., \& Houdijk, H. (2013). External attentional focus enhances movement automization: A comprehensive test of the constraint action hypothesis. Human Movement Science, 32, 527-539. https://doi.org/10.1016/j.humov.2013.04.001.

Kleynen, M., Braun, S. M., Rasquin, S. M. C., Bleijlevens, M. H. C., Lexis, M. A. S., Halfens, J., ... Beurskens, A. J. (2015). Multidisciplinary views on applying explicit and implicit motor learning in practice: An international survey. PLoS One, 1O(8), e0135522. https://doi.org/10.1371/journal.pone.0135522.

Krajenbrink, H., van Abswoude, F., Vermeulen, S., van Cappellen, S., \& Steenbergen, B. (2018). Motor learning and movement automatization in typically developing children: The role of instructions with an external or internal focus of attention. Human Movement Science, 60, 183-190. https://doi.org/10.1016/j.humov.2018. 06.010.

Masters, R. S. W. (1992). Knowledge, knerves, and know-how: The role of explicit versus implicit knowledge in the breakdown of a complex motor skill under pressure. British Journal of Psychology, 83, 343-358. https://doi.org/10.1111/j.2044-8295.1992.tb02446.x.

Masters, R. S. W., \& Poolton, J. M. (2012). Advances in implicit motor learning. In N. J. Hodges, \& A. M. Williams (Eds.). Skill acquisition in sport: Research, Theory and Practice (pp. 59-75). (2nd ed.). London: Routledge.

Maxwell, J. P., Masters, R. S. W., \& Eves, F. F. (2003). The role of working memory in motor learning and performance. Consciousness and Cognition, 12(3), 376-402. https://doi.org/10.1016/S1053-8100(03)00005-9.

Maurer, H., \& Munzert, J. (2013). Influence of attentional focus on skilled motor performance: Performance decrement under unfamiliar focus conditions. Human Movement Science, 32, 730-740. https://doi.org/10.1016/j.humov.2013.02.001.

Perreault, M. E., \& French, K. E. (2016). Differences in children's thinking and learning during attentional focus instruction. Human Movement Science, 45(Supplement C), 154-160. https://doi.org/10.1016/j.humov.2015.11.013.

Piek, J. P., Dyck, M. J., Francis, M., \& Conwell, A. (2007). Working memory, processing speed, and set-shifting in children with developmental coordination disorder and attention-deficit-hyperactivity-disorder. Developmental Medicine and Child Neurology, 49, 678-683. https://doi.org/10.1111/j.1469-8749.2007.00678.x.

Poolton, J. M., Maxwell, J. P., Masters, R. S. W., \& Raab, M. (2006). Benefits of an external focus of attention: Common coding or conscious processing? Journal of Sports Sciences, 24(1), 89-99. https://doi.org/10.1080/02640410500130854.

Quinn, J. G. (2008). Movement and visual coding: The structure of visuo-spatial working memory. Cognitive Processing, 9(1), 35-43. https://doi.org/10.1007/s10339007-0184-9.

Saemi, E., Porter, J., Wulf, G., Ghotbi-Varzaneh, A., \& Bakhtiari, S. (2013). Adopting an external focus of attention facilitates motor learning in children with attention deficit hyperactivity disorder. Kineziologija, 45(2), 179-185.

Schoemaker, M. M., \& Smits-Engelsman, B. C. M. (2015). Is treating motor problems in DCD just a matter of practice and more practice? Current Developmental Disorders Reports, 2(2), 150-156. https://doi.org/10.1007/s40474-015-0045-7.

Smits-Engelsman, B. C. M., Blank, R., Van Der Kaay, A. C., Mosterd-Van Der Meijs, R., Vlugt-Van Den Brand, E., Polatajko, H. J., \& Wilson, P. H. (2013). Efficacy of interventions to improve motor performance in children with developmental coordination disorder: A combined systematic review and meta-analysis. Developmental Medicine and Child Neurology, 55(3), 229-237. https://doi.org/10.1111/dmcn.12008.

Steenbergen, B., van der Kamp, J., Verneau, M., Jongbloed-Pereboom, M., \& Masters, R. S. W. (2010). Implicit and explicit learning: Applications from basic research to sports for individuals with impaired movement dynamics. Disability and Rehabilitation, 32(18), 1509-1516. https://doi.org/10.3109/09638288.2010.497035.

Thorn, J. E. (2006). Using attentional strategies for balance performance and learning in nine through 12 year olds. Doctoral dissertationTallahassee: Florida State University.

van Abswoude, F., Nuijen, N. B., van der Kamp, J., \& Steenbergen, B. (2018). Individual differences influencing immediate effects of internal and external focus instructions on children's motor performance. Research Quarterly for Exercise and Sport, 1-10. https://doi.org/10.1080/02701367.2018.1442915.

van Abswoude, F., van der Kamp, J., \& Steenbergen, B. (2018). The roles of declarative knowledge and working memory in explicit motor learning and practice among children with low motor abilities. Advance online publication Motor Control. https://doi.org/10.1123/mc.2017-0060.

Wilson, P. H., Ruddock, S., Smits-Engelsman, B., Polatajko, H., \& Blank, R. (2013). Understanding performance deficits in developmental coordination disorder: A meta-analysis of recent research. Developmental Medicine and Child Neurology, 55, 217-228. https://doi.org/10.1111/j.1469-8749.2012.04436.x.

Wilson, P. H., Smits-Engelsman, B., Caeyenberghs, K., Steenbergen, B., Sugden, D., Clark, J., ... Blank, R. (2017). Cognitive and neuroimaging findings in developmental coordination disorder: New insights from a systematic review of recent research. Developmental Medicine and Child Neurology, 59(11), 1117-1129. https:// doi-org.ru.idm.oclc.org/10.1111/dmen.13530.

Wulf, G. (2013). Attentional focus and motor learning: A review of 15 years. International Review of Sport and Exercise Psychology, 6(1), 77-104. https://doi.org/10. 1080/1750984x.2012.723728.

Wulf, G., Chiviacowsky, S., Schiller, E., \& Ávila, L. T. G. (2010). Frequent external-focus feedback enhances motor learning. Frontiers in Psychology, 1, 190. https://doi. org/10.3389/fpsyg.2010.00190.

Wulf, G., McNevin, N., \& Shea, C. H. (2001). The automaticity of complex motor skill learning as a function of attentional focus. The Quarterly Journal of Experimental Psychology A, 54, 1143-1154. https://doi.org/10.1080/02724980143000118.

Wulf, G., \& Prinz, W. (2001). Directing attention to movement effects enhances learning: A review. Psychonomic Bulletin and Review, 8(4), 648-660. https://doi.org/10. 3758/BF03196201.

Zwicker, J. G., Harris, S. R., \& Klassen, A. F. (2013). Quality of life domains affected in children with developmental coordination disorder: A systematic review. Child: Care, Health and Development, 39(4), 562-580. https://doi.org/10.1111/j.1365-2214.2012.01379.x. 